\title{
Bayesian methods for mixed method synthesis
}

\section{Leonardo Roever*}

Department of Clinical Research, Federal University of Uberlândia, Brazil

Bayesian methods generate summative statements of the evidence through the meta-aggregation of data, and this can involve attributing a numerical value to all qualitative data, facilitating a final statistical analysis of individual syntheses (i.e. translating qualitative data into quantitative), or attributing a qualitative thematic description to all quantitative data, thereby permitting a final meta-aggregation of individual syntheses (i.e. translating quantitative data into qualitative) (Table 1) [1-4].

The Bayesian method of data analysis can be used to summarize uncertainties and make estimates and predictions using probabilistic statements conditioned by the observation of data in an assumed model. The Bayesian approach to statistical inference has the characteristic of considering the unknown parameters of the models as random quantities, and thus expresses the uncertainty about these parameters through a priori distributions of probabilities. Through the use of the Bayes' theorem, the updating of the uncertainty about the parameters can be done sequentially with the arrival of new information [1-4].

The Bayesian approach more comfortably accommodates metaanalysis techniques where uncertainty about the effect of a treatment and can be updated sequentially with the execution of another study on the subject, this allows more flexible models where individual

Table 1. Comparison of Bayesian methods for mixed method synthesis

\begin{tabular}{|c|c|}
\hline Method of synthesis & Description \\
\hline $\begin{array}{l}\text { Bayesian conversion: } \\
\text { Qualitative } \rightarrow \text { Quantitative }\end{array}$ & $\begin{array}{l}\text { A numerical value is attributed to all qualitative data in a } \\
\text { format that is complementary to that of the quantitative } \\
\text { data; } \\
\text { Separate prior distributions (presumed probabilities) are } \\
\text { applied individually to quantitative and qualitative data; } \\
\text { Posterior distributions (evidence informed probabilities) } \\
\text { are compared and, if } 95 \% \text { confidence intervals overlap, } \\
\text { combined. }\end{array}$ \\
\hline Strengths & $\begin{array}{l}\text { Facilitates the statistical analysis of both quantitative and } \\
\text { qualitative data; } \\
\text { Attributes equal strength to quantitative and qualitative } \\
\text { data instead of simply using qualitative data for the } \\
\text { generation of themes in which to impute quantitative data. }\end{array}$ \\
\hline Weaknesses & $\begin{array}{l}\text { Significant problems associated with conversion of verbal } \\
\text { counts (e.g. "many", "few" etc) to numerical format; } \\
\text { Non-overlapping } 95 \% \text { confidence intervals prohibit } \\
\text { combined analysis of data. }\end{array}$ \\
\hline $\begin{array}{l}\text { Bayesian conversion: } \\
\text { Quantitative } \rightarrow \text { Qualitative }\end{array}$ & $\begin{array}{l}\text { All quantitative data is thematically synthesized and } \\
\text { codified according to strength of effect. }\end{array}$ \\
\hline Strengths & $\begin{array}{l}\text { Codification of quantitative data is less error prone than } \\
\text { quantification of qualitative data; } \\
\text { Existing data provides a more accurate basis for the } \\
\text { development of prior distributions through which to base } \\
\text { subsequent analyses. }\end{array}$ \\
\hline Weaknesses & $\begin{array}{l}\text { Whole study focus produces a reliance on reasonable } \\
\text { number of studies for strength of conclusions; } \\
\text { The weighting of individual studies needs to be } \\
\text { adjusted based on the varying levels of evidence and } \\
\text { methodological quality of these included studies. }\end{array}$ \\
\hline
\end{tabular}

characteristics of the studies can be easily incorporated. The Bayesian approach has as a principle to combine the external information to the studies, defining an a priori distribution for the meta-analytical measurement and for the variability between the studies and incorporate them to the analysis [1-4].

Bayesian inference is an alternative method to classical inference that allows incorporating a priori information about the parameters of the model. Bayesian methods consider the parameters of the models as random quantities assigning probability distributions to them (a priori and a posteriori distributions), that is, Bayesian methods incorporate information extraneous to the data of the experiment on the parameters through priories [1-4].

This information is combined with the information obtained from the analysis data of the experiments through Bayes' theorem and the inferences are based on the posterior distributions [1-5].

In conclusion, Bayesian methods can improve the accuracy and interpretation of study results.

\section{References}

1. Alan P, White H, Bath-Hextall F, Salmond S, Apostolo J, et al. (2015) A mixedmethods approach to systematic reviews. Int J Evid Based Health 13: 121-31.

2. Sandelowski M, Voils CI, Barroso J (2006) Defining and Designing Mixed Research Synthesis Studies. Res Sch 13: 29.

3. Robert CP (2010) On the relevance of the Bayesian approach to statistics. Review of economic analysis 2 : 139-152.

4. Pearson A (2016) Meta-aggregation: emergence of the "missing" piece in qualitative synthesis. JBI Database System Rev Implement Rep 14: 1-3.

5. Crandell JL, Voils CI, Chang Y, Sandelowski M (2011) Bayesian data augmentation methods for the synthesis of qualitative and quantitative research findings. Qual Quant 45: $653-69$

Copyright: (C2019 Roever L. This is an open-access article distributed under the terms of the Creative Commons Attribution License, which permits unrestricted use, distribution, and reproduction in any medium, provided the original author and source are credited.

${ }^{\star}$ Correspondence to: Leonardo Roever, MHS, PhD, Department of Clinical Research, Av. Pará, 1720 - Bairro Umuarama, Uberlândia - MG - CEP 38400902, Brazil, Tel: +553488039878; E-mail: leonardoroever@hotmail.com

Received: July 09, 2019; Accepted: August 02, 2019; Published: August 06, 2019 